\title{
Gestão de institutos públicos de pesquisa no Brasil: limites do modelo jurídico
}

\author{
Valéria Cristina dos Santos Ribeiro \\ Instituto Nacional de Pesquisas Espaciais (Inpe) \\ São José dos Campos / SP - Brasil \\ Sergio Luiz Monteiro Salles-Filho \\ Universidade Estadual de Campinas (Unicamp) / Departamento de Política Científica e \\ Tecnológica, Instituto de Geociências \\ Campinas / SP - Brasil \\ Adriana Bin \\ Universidade Estadual de Campinas (Unicamp) / Faculdade de Ciências Aplicadas \\ Campinas / SP - Brasil
}

\begin{abstract}
Este trabalho tem como objetivo identificar quais os espaços de autonomia e flexibilidade gerencial e organizacional dos institutos públicos de pesquisa (IPPs) no Brasil em face dos limites impostos por seu arcabouço legal, assim como os desdobramentos dessas condições para o cumprimento e o desempenho das missões institucionais. Para tal, foram buscadas experiências distintas sobre reorganizações organizacionais, modelos jurídicos e legislações de IPPs no Brasil e no mundo, e realizados dois estudos de caso com IPPs brasileiros - Instituto Nacional de Pesquisas Espaciais (Inpe) e Centro de Tecnologia da Informação Renato Archer (CTI) —, ambos sujeitos ao regime jurídico da administração direta federal. Como conclusão destaca-se a existência de graus de liberdade gerencial que abrem perspectivas de autonomia e flexibilidade que podem ser aproveitadas de forma variada e ampliada por parte desse tipo de organização.
\end{abstract}

Palavras-chave: gestão de P\&D; governança da pesquisa; modelos jurídicos; institutos públicos de pesquisa.

Gestión de los institutos públicos de investigación en Brasil: límites del modelo legal Este trabajo tiene como objetivo identificar los espacios de autonomía y flexibilidad gerencial y organizacional de los institutos públicos de investigación (IPIs) en el Brasil frente a los límites impuestos por la legislación y las consecuencias de estas condiciones para la conducción de sus misiones institucionales. Para tal fin, fueron seleccionadas distintas experiencias sobre la reestructuración organizacional, modelos jurídicos y la legislación de IPIs en el Brasil y en el mundo, realizando también dos estudios de caso con IPIs brasileños - el Instituto Nacional de Investigaciones Espaciales (Inpe) y el Centro de

DOI: http://dx.doi.org/10.1590/0034-7612126590

Artigo recebido em 14 nov. 2013 e aceito em 12 dez. 2014.

(cc) BY-NC 
Tecnología de la Información Renato Archer (CTI) - , ambas instituciones que operan bajo el régimen jurídico de la Administración Directa Federal. Como conclusión, se destaca la existencia de grados de libertad para los modelos de gestión, que les abren perspectivas de autonomía y flexibilidad que pueden ser aprovechadas de forma variada y ampliada por parte de ese tipo de organización.

Palabras clave: gestión de I+D; modelos de gobierno de la investigación; modelos jurídicos; institutos públicos de investigación.

Management of public research institutes in Brazil: limits of the legal framework

This article aims to identify what are the autonomy spaces and the organizational and managing flexibility of public research organizations in Brazil, given the limits imposed by its legal framework and the consequences for the accomplishment and performance of institutional missions. In order to do it, different experiences on organizational restructuring, legal models and legislation of public research institutes in Brazil and around the world were analyzed, and two case studies with Brazilian public research institutes were presented - the National Institute of Space Research (Inpe) and the National Center for Information Technology Renato Archer (CTI) - , both belonging to the federal administration and governed by the same legal framework. The conclusions point out for the existence of degrees of managing freedom, which bring perspectives of flexibility and autonomy that could be used and explored in different ways and expanded by this kind of organization.

KEYWORDs: R\&D management; governance of R\&D; legal framework; public research organizations.

\section{Introdução}

Este artigo tem por objetivo apresentar resultados de uma pesquisa sobre modelos gerenciais de Institutos Públicos de Pesquisa (IPPs) da Administração Direta Federal (ADF) no Brasil, abordando três questões principais: (i) em que medida os IPPs da ADF são efetivamente limitados em suas ações e capacidades gerenciais por conta de modelos jurídicos restritivos?; (ii) quais os tipos e graus de autonomia que existem para esses institutos e como o exercício dessa autonomia pode criar ações gerenciais diferenciadas - e mais ou menos exitosas sob um mesmo regime jurídico?; (iii) é preciso criar regimes jurídicos alternativos aos existentes no cenário brasileiro para que os institutos de pesquisa cumpram melhor suas missões? Essas questões são recorrentes na literatura e vêm sendo debatidas em praticamente todos os fóruns que tratam do presente e do futuro das organizações públicas de pesquisa.

A questão do modelo jurídico da pesquisa pública remonta aos anos $1970 .{ }^{1}$ Já no final dessa década e no início dos anos 1980 alguns autores fizeram referência à necessidade de buscar um arcabouço legal mais flexível para as atividades de pesquisa e desenvolvimento (P\&D). Albuquerque e colaboradores (1986), discutindo o caso do Instituto Agronômico de Campinas, mostravam que vários elementos de desempenho operacional daquele instituto estavam diretamente ligados ao seu modelo jurídico de baixa autonomia e flexibilidade.

\footnotetext{
${ }^{1}$ Neste artigo entende-se por modelo jurídico de uma organização as características formais dentro das quais ela é legalmente constituída.
} 
Da mesma forma, Alves (1985) mostrou a importância do modelo empresarial adotado pela Empresa Brasileira de Pesquisa Agropecuária (Embrapa), mas já alertava que, mesmo sob um regime jurídico mais flexível das empresas públicas, o instituto vinha perdendo autonomia na execução de sua missão.

Ao longo dos anos 1980 e 1990 o debate prosseguiu. Salles-Filho e colaboradores (2000) fizeram uma revisão desse tema, mostrando que as amarras legais já colocavam em risco a competitividade dessas organizações em face de um movimento de diversificação dos atores dos sistemas de inovação (SI). Mais recentemente, Madi e colaboradores (2006) propuseram uma dupla agenda para o futuro das instituições públicas de pesquisa agrícola do estado de São Paulo, sendo uma delas exatamente a da modernização do marco legal no qual operam essas organizações.

A publicação do CGEE (2010) faz uma discussão ampliada sobre os modelos institucionais de organizações de pesquisa no Brasil com cerca de 200 organizações desse tipo, divididas em três categorias: administração pública, entidades empresariais e entidades sem fins lucrativos. O estudo encontrou 17 diferentes modelos jurídicos, com variados graus de autonomia de gestão. As limitações que mais penalizavam a agilidade na realização de contratos de bens e serviços e na contratação de recursos humanos estavam na esfera da administração pública, particularmente da ADF.

Nos últimos anos, leis e decretos têm sido publicados com o intuito de fomentar o desenvolvimento e a relação entre universidades e institutos de pesquisa com fundações de apoio e empresas. Entre essas legislações encontram-se a Lei no $12.349 / 2010$, que alterou pontos da Lei de Licitações, favorecendo o desenvolvimento e a inovação tecnológica realizados no país; a Lei ํo 12.863/2013, que alterou a carreira do Magistério Federal e facilitou as relações de docentes com o sistema de inovação; além da PEC no 290/2013, que atualizou o tratamento das atividades de ciência, tecnologia e inovação nos dispositivos constitucionais. Deve-se ainda registrar a importância da legislação de propriedade industrial (Lei no 9.279/1996), que consolidou um marco regulatório mais claro para a definição dos direitos de propriedade de bens intangíveis com impactos nas relações de IPPs com empresas e mesmo com seus próprios pesquisadores.

O marco legal mais abrangente que está atualmente em discussão no Congresso Nacional é do Projeto de Lei ํㅡ $2.177 / 2011$. Trata-se de um novo arcabouço jurídico para regular as organizações e atividades de P\&D no Brasil, baseado na criação do Código Nacional de Ciência, Tecnologia e Inovação, que substituiria diversas leis, inclusive a própria Lei de Inovação (Lei $\mathrm{n}^{\mathrm{o}}$ 10.973/2004). Entre outros objetivos, procura dar maior flexibilidade operacional e espaço gerencial para as organizações de pesquisa. ${ }^{2}$

\footnotetext{
${ }^{2}$ Sem entrar na proposição de um novo modelo institucional (e jurídico) para os IPPs, o PL no 2.177/2011, ainda em discussão, aborda o tema das compras públicas para Ciência e Tecnologia e Inovação, propondo um processo mais ágil de contratações intitulado "Seleção Simplificada", que tenta resolver um dos pontos mais difíceis da gestão da pesquisa pública.
} 
Modelos de maior autonomia e flexibilidade já existem no Brasil nos IPPs que se qualificaram como Organizações Sociais (OS), que operam por meio de um Contrato de Gestão com órgãos de governo. Os tempos e movimentos gerenciais tendem a ser muito mais ágeis em OS, como mostram Salles-Filho e colaboradores (2000) e Brum e Meneghini (2002).

Assim, nos últimos 30 anos, os IPPs — não apenas nacionais, mas também de outros países - vêm enfrentando vários desafios relacionados à adequação de seus modelos jurídicos, assim como de seus modelos gerenciais e organizacionais, ${ }^{3}$ para sua efetiva inserção nos SI. Estes desafios têm se materializado em várias iniciativas de reorganização, como as descritas por Jain e Triandis (1997), Senker (2000), Poti e Reale (2000), Salles-Filho e colaboradores (2000), Quental e colaboradores (2001), Prest (2002), Boden e colaboradores (2006), SallesFilho e Bonacelli (2010).

Muito embora a busca de novos modelos jurídicos, evidenciada a partir de experiências dos países, venha no intuito de ampliar eficiência, efetividade e autonomia dos IPPs, a relação entre esses três aspectos não se coloca de forma clara, uma vez que há alterações jurídicas importantes que não são acompanhadas por alterações gerenciais que levem a maior eficiência e efetividade e, ao mesmo tempo, há alterações gerenciais que são implantadas de forma independente do modelo jurídico e que trazem impactos positivos.

Boden e colaboradores (2006) analisaram a reorganização dos IPPs ingleses nas últimas décadas e concluíram que o impacto dos novos modelos jurídicos sobre a eficiência e efetividade dessas organizações não é claro e que a razão básica para a ocorrência das mudanças (especialmente no caso das privatizações) foi muito mais política (e exógena) do que baseada em uma racionalidade gerencial.

Modelos alternativos aos da administração pública direta, como os Cooperative Research Centres australianos (Slatyer, 1994), os institutos privados sem fins lucrativos que atuam como empresas de serviços, como o Instituto Fraunhofer da Alemanha, ou as organizações sociais no Brasil, vêm sendo buscados desde o início dos anos 1990 como formatos mais ágeis e flexíveis (e competitivos).

Em linhas gerais, os modelos de gestão de um IPP são limitados por dois tipos principais de restrições: as restrições legais associadas com o modelo jurídico do setor público, no qual prevalece a legalidade estrita (uma organização pública só pode fazer o que a lei autoriza que faça), e a influência política à qual é submetida, que em linhas gerais seria ditada pela maior ou menor atuação do governo sobre sua missão, atuação e desempenho (Arnold, 2007; Jansen, 2007; Garcia e Salles-Filho, 2009).

\footnotetext{
${ }^{3}$ Modelo gerencial são as formas estratégicas, táticas e operacionais pelas quais a instituição se organiza e conduz seus processos básicos de gestão dentro de um dado modelo jurídico, isto é, a organização das atividades-fim e meio e a condução dos seus fluxos e processos decisórios. O modelo gerencial também é composto pelo chamado modelo organizacional, que se refere a divisão do trabalho, atribuição de responsabilidades e autoridade, comunicação e coordenação e que se reflete no organograma da instituição. Normalmente, o modelo de gestão adotado define qual será o modelo organizacional.
} 
Nesse contexto, este artigo propõe-se a identificar quais os espaços de autonomia e flexibilidade gerencial e organizacional dos IPPs em face dos limites impostos por seu arcabouço legal. Para tal, foram buscadas experiências nacionais e internacionais e realizados estudos de caso com dois IPPs brasileiros: o Instituto Nacional de Pesquisas Espaciais (Inpe) e o Centro de Tecnologia da Informação Renato Archer (CTI), ambos da ADF.

O tema das relações entre o arcabouço jurídico normativo do setor público e os espaços e limites gerenciais de uma organização de ciência e tecnologia, embora presente nos debates sobre papéis e estratégias de organizações públicas de pesquisa, como observado por Senker (2000), carece de estudos que mostrem em que medida as limitações legais realmente impedem (ou estimulam) essas organizações a cumprirem suas missões.

Muitas vezes, toda a carga de problemas gerenciais é atribuída ao modelo jurídico e suas restrições. Ainda que limitações existam, o presente artigo contribui para compreender a forma, o grau e a direção dessas limitações. Nesse sentido, o trabalho ilumina aspectos ainda não analisados comparativamente em IPPs brasileiros, particularmente por meio do estudo de presença ou ausência de práticas gerenciais que permitem realizar atividades cruciais para essas organizações apesar dos limites legais restritivos da ADF.

O artigo está estruturado em quatro seções, incluindo a presente introdução. A próxima seção identifica, por meio de ampla revisão de literatura, os principais temas de análise dos espaços gerenciais e organizacionais (nominalmente, buscou-se, além de literatura acadêmica,

material sobre trajetórias de IPPs nos Estados Unidos, União Europeia, China, Índia e Coreia — e também no Brasil). No processo de escolha dos países buscou-se identificar experiências distintas em termos de localização e nível de desenvolvimento, com vistas a captar diversidade de situações.

A terceira seção apresenta em linhas gerais as características de organizações da ADF, seguida dos resultados dos estudos do Inpe e do CTI, ambos sujeitos ao mesmo regime jurídico, mas com importantes variações em termos gerenciais e organizacionais. Finalmente, a última seção apresenta as conclusões do trabalho. A metodologia é descrita dentro de cada seção, sempre que necessário.

\section{Identificação dos principais temas gerenciais e organizacionais nas trajetórias de IPPs}

A organização dos IPPs em âmbito global, embora com diferenças entre os países, ocorreu, grosso modo, em duas fases. A primeira entre os anos 1980 e 1990 e a segunda a partir dos anos 2000. Na primeira fase, as mudanças nos IPPs foram motivadas por alguns fatores principais, tais como: crise fiscal e financeira e transformações nos papéis do Estado (incluindo restrições em termos de recursos financeiros), surgimento de novos campos do conhecimento, com transformações técnico-científicas, e, por fim, emergência de novos atores e de novas relações entre eles no âmbito dos sistemas de pesquisa e de inovação (Salles-Filho et al., 2000; Oecd, 2003; Laredo e Mustar, 2004; Furtado, 2005; Salles-Filho e Bonacelli, 2010). Coccia e Rolfo (2008), 
analisando vários países da Europa, relatam que os IPPs procuraram se diversificar por meio da venda de serviços, alavancagem de financiamento e uma estratégia mais focada na sobrevivência do que na evolução madura em direção a novos papéis nos sistemas de inovação.

No decorrer dos anos 2000, passada a primeira fase de mudanças decorrentes das questões abordadas, ocorreu (e ainda está ocorrendo) uma nova fase de transformações. Bin e colaboradores (2013) mostram, por meio de estudo comparativo entre IPPs agrícolas do Brasil, EUA, Canadá e Uruguai, que a tendência mais forte está justamente na busca por novas formas de inserção dos institutos nos respectivos SI.

A revisão de literatura permitiu a identificação de cinco temas recorrentes, que ajudam a entender os espaços gerenciais que vêm sendo desenvolvidos por IPPs neste último período. Esses temas são: governança institucional; ciclo completo do planejamento; manutenção e ampliação de recursos humanos; captação e gestão de recursos financeiros; e relacionamento com atores dos SI. A seguir apresentam-se, de forma resumida, os conteúdos de cada um desses temas.

Os elementos que compõem o tema da governança institucional são estudados por vários autores, entre eles Laredo e Mustar (2004), Jansen (2007), Âström e colaboradores (2008), Garcia e Salles-Filho (2009), Adler e colaboradores (2009), Hollanders e Soete (2010), Mattos e Abdal (2010) e Bin e colaboradores (2013), além de organizações, como Ocde (2011). Esses autores e organizações têm demonstrado que a busca por boas práticas de governança aparece como um dos elementos centrais das trajetórias recentes de IPPs, com franca tendência à criação de modelos de governança baseados em transparência e em interfaces de comunicação internas e externas. Seus trabalhos abordam questões como instâncias de representação internas e externas como conselhos e comitês; modelos de organização da direção, mais ou menos participativos; inovações organizacionais; profissionalização da comunicação institucional; e práticas de prestação de contas.

A sistematização do ciclo completo do planejamento, envolvendo programação, monitoramento e avaliação, é outro componente que aparece nos estudos sobre o tema dos IPPs. Autores como Arnold e colaboradores (1998), Âström e colaboradores (2008), Hollanders e Soete (2010), Salles-Filho e Bonacelli (2010) e Bin e colaboradores (2013) e organizações como Ocde (2003) apontam que o planejamento tornou-se imprescindível aos IPPs de todas as áreas do conhecimento, inclusive em áreas do conhecimento que trabalham com pesquisa básica e prezam o modelo blue sky research, que sistematizam ações de planejamento para poder competir por recursos humanos, materiais e financeiros.

Com relação à manutenção e ampliação de recursos humanos, Senker (2000), Salles-Filho e colaboradores (2000), Laredo e Mustar (2004), Âström e colaboradores (2008) e Ocde (2011) abordam questões sobre as formas de contratação dos recursos humanos, o treinamento e o desenvolvimento da mão de obra especializada, a implantação de sistemas de avaliação e premiação e a busca por cooperação com outras organizações para ampliar massa crítica em projetos e programas específicos. Outro ponto comum é a internacionalização do mercado de trabalho: contratações de estrangeiros por organizações nacionais aparecem como um dos elementos da internacionalização dos IPPs (Ocde, 2011).

Com relação aos recursos financeiros, a trajetória dos IPPs foi claramente a de capacitação para um ambiente cada vez mais competitivo. Arnold e colaboradores (1998), Salles-Filho 
e colaboradores (2000), Laredo e Mustar (2004), Âström e colaboradores (2008), Hollanders e Soete (2010) e Ocde (2011) mostram justamente essa tendência: recursos diretos dos governos são cada vez mais complementados por recursos competitivos, levando a um movimento de profissionalização de competências e à definição de diretrizes internas com metas de captação de recursos financeiros.

Finalmente, o tema do relacionamento com os atores dos sistemas de inovação complementa os temas aqui propostos para a análise dos espaços gerenciais. Senker (2000), Hall (2006), Âström e colaboradores (2008), Mattos e Abdal (2010), Dossa e Segatto (2010), Hollanders e Soete (2010), Ocde (2011) e Bin e colaboradores (2013) apontam os mais variados mecanismos introduzidos pelos IPPs para ampliar sua inserção nos SI por meio da ampliação de seu relacionamento com outras organizações de pesquisa, governos e, principalmente, empresas. Desde o final dos anos 1990 e início da década de 2000 esse já era um fenômeno recorrente (Vonortas, 2000; Ocde, 2003).

\section{Espaços gerenciais e organizacionais sob mesmo regime jurídico: casos do Inpe e do CTI}

A contribuição do presente artigo está na identificação de espaços de autonomia e flexibilidade gerencial e organizacional que podem ser explorados de diversas maneiras pelos IPPs, mesmo sob restrições legais dos modelos jurídicos da ADF. Antes de entrarmos nos casos estudados, é preciso apresentar as características do regime jurídico da ADF e sua importância para as questões gerenciais e organizacionais dos IPPs.

\subsection{Administração direta federal: quais as implicações para os IPPs?}

No Brasil, existem diversos modelos jurídicos para os IPPs, com destaque para administração direta, administração indireta (como autarquia, fundação pública, empresa pública) e Organização Social (OS). Uma análise conjunta destes modelos feita por CGEE (2010) identificou 17 diferentes modelos e apontou o modelo de ADF como o mais restritivo.

Os órgãos da ADF são unidades de atuação do Estado desprovidas de personalidade jurídica própria (não se apresentam como sujeitos de direitos e obrigações, por si próprios), estando inseridas na estrutura de outra organização, esta sim provida de personalidade jurídica. Podem firmar Termo de Compromisso de Gestão (TCG) junto ao órgão hierárquico superior (o que ocorre de fato com os institutos vinculados ao MCTI), que nada mais é do que uma versão fraca do instrumento de contrato de gestão celebrado entre o governo e as OS. ${ }^{4}$

\footnotetext{
${ }^{4} \mathrm{O}$ contrato de gestão está previsto no artigo 37, §8ํㅡ, da Constituição Federal (Rosa, 2007; Meirelles et al., 2012).
} 
Como todo organismo governamental no Brasil, os IPPs da ADF estão submetidos à Lei no 8.666/1993 — Lei sobre Licitações e Contratos —, que regulamenta toda forma de compra, alienação (venda) e contratação de serviços, assim como à Lei no 8.112/1990, relacionada com a administração de pessoas.

As principais limitações impostas aos IPPs em função da legislação que rege a administração direta estão relacionadas com a gestão orçamentária e financeira e de recursos humanos. Sobre o primeiro ponto, além dos recursos advindos do orçamento da União (para os quais essas restrições existem), os IPPs federais têm liberdade para buscar recursos em outras fontes, movimento que se tornou cada vez mais necessário a partir de restrições orçamentárias do Tesouro e a partir de um processo cada vez mais competitivo do financiamento da pesquisa.

Para a captação de recursos em decorrência da comercialização de produtos e serviços, os IPPs podem se utilizar de fundações e outras estruturas jurídicas que na prática intermedeiam contratos de forma a tornar mais ágil sua execução. Entretanto, mais recentemente o TCU tem restringido esse mecanismo por meio de interpretações que, na prática, aproximam os procedimentos de órgãos da administração direta aos de entidades que intermedeiam e apoiam os IPPs, como as fundações de apoio.

No que se refere à gestão de recursos humanos, as principais questões referem-se à contratação, que deve ser via concurso público mediante a existência de vagas definidas no âmbito do Ministério do Planejamento, Orçamento e Gestão (MPOG). Completa esse quadro a baixa flexibilidade na definição de planos de carreira e desenvolvimento.

Outro ponto crítico para esses IPPs é a relativa baixa autonomia e flexibilidade para estabelecer e gerenciar contratos, em razão de serem figuras jurídicas despersonalizadas. Para minimizar essa condição, os IPPs podem também se valer (assim como no caso da venda de produtos e serviços) de intervenientes administrativos, como as fundações de apoio.

No que se refere aos mecanismos de planejamento, programação e avaliação das atividades dos institutos, são poucas as limitações impostas pelo modelo. Apesar de o MCTI apresentar uma sugestão para o formato de realização do planejamento dos IPPs, há suficiente liberdade para que os institutos decidam internamente como o planejamento efetivamente se dará (se será participativo ou não, se será contratada uma consultoria de apoio, se incluirão outras vertentes além daquelas indicadas pelo Ministério, quais metodologias e ferramentas serão empregadas).

Com relação à avaliação, ocorre o mesmo. Além dos indicadores pactuados junto ao MCTI e no TCG, os institutos podem incluir outros indicadores que julgarem pertinentes para gerenciamento de suas atividades e mensuração de seus resultados e impactos, tanto do ponto de vista interno quanto para a divulgação para a sociedade.

Ainda nessa dimensão, destacam-se a autonomia e flexibilidade dos IPPs no que se refere à definição das formas de organização da pesquisa, gestão de portfólios, programas e projetos, gestão de parcerias e redes de cooperação nacionais e internacionais, gestão da propriedade intelectual e transferência de tecnologia e gestão do conhecimento. 
Observada a restrição do número de gratificações de chefias (Direção e Assessoramento Superior - DAS) e das denominações de suas unidades organizacionais, os IPPs podem também definir o tipo de modelo organizacional que terão - se divisional, matricial, funcional, ou orgânico. O formato de escolha das chefias internas também é uma decisão interna do IPP (comitê de busca, eleição ou indicação de pessoas escolhidas pelo diretor, que podem ser internas ou externas). Por fim, os institutos também têm, nesse modelo, liberdade para definir suas políticas e práticas de comunicação institucional.

\subsection{Análise comparada dos modelos gerenciais do Inpe e CTI}

Esta seção apresenta os resultados da análise comparativa das iniciativas gerenciais dos IPPs selecionados, discutindo quais são as iniciativas de aproveitamento de espaços de autonomia e flexibilidade gerencial que existem no contexto dos limites impostos pelo modelo jurídico ao qual estão sujeitos.

Os institutos escolhidos foram o Inpe e o CTI, duas instituições federais da administração direta pertencentes ao MCTI. Ambos têm missões focadas em setores de alta tecnologia, que apresentam forte demanda por P\&D e por inovação (setor espacial e de tecnologia de informação e comunicação), e têm por objetivo interagir com atores do SI, particularmente empresas e governo. Ademais, ambos estão situados em polos tecnológicos importantes do estado de São Paulo, apresentando similitudes também no contexto geográfico social. ${ }^{5}$

A metodologia empregada foi qualitativa, partindo de uma ampla revisão de literatura sobre reorganização institucional de IPPs no Brasil e em âmbito internacional, e fundandose em estudos de caso complementados por análise documental. Segundo Godoy (1995a, 1995b), essas duas abordagens, juntamente com a etnografia, representariam os três principais conjuntos de métodos clássicos da pesquisa qualitativa com foco em administração.

No presente trabalho, buscaram-se informações para colher evidências sobre como os espaços de gestão são diferenciados dentro de modelos jurídicos reconhecidamente restritivos. Como aponta Pereira (1999), a pesquisa qualitativa, seja por meio da quantificação ou não das informações e evidências colhidas, permite esclarecer aquilo que muitas vezes é genericamente observado, mas ainda não empiricamente demonstrado.

O questionário semiestruturado foi preparado com base nos cinco temas mencionados, procurando obter evidências das práticas e modelos de gestão adotados ou desenvolvidos nos IPPs estudados.

Apesar de não se tratar de uma pesquisa abrangente, do tipo survey, optou-se por testar o questionário para aprimorá-lo antes da realização das entrevistas e de seu preenchimento. Foram então realizados pré-testes com pesquisadores de diferentes organizações de pesquisa

\footnotetext{
${ }^{5}$ Experiências de trabalhos anteriores junto aos dois IPPs por parte dos autores deste artigo, somadas às facilidades de acesso pela proximidade geográfica dos dois IPPs, foram fatores complementares para sua escolha como casos.
} 
brasileiras (além dos dois IPPs-alvos da pesquisa, também foram consultados pesquisadores da Embrapa, ITA/DCTA, Ipea e CGEE). Esses pré-testes foram importantes para calibrar três pontos: clareza e desambiguação das questões e das estruturas de resposta; pertinência e alcance das questões para responder aos objetivos da pesquisa; eliminação de redundâncias e sobreposições sem funções de verificação lógica.

O questionário foi aplicado presencialmente no ano de 2013 e buscou informações e dados referentes ao período de 2003 a 2012. Todos os entrevistados são servidores de carreira que se encontram nos institutos por pelo menos 15 anos. Ao todo foram realizadas oito entrevistas (quatro em cada Instituto), incluindo os respectivos diretores. A análise documental sobre os IPPs estudados precedeu tanto a elaboração do questionário como a realização das entrevistas.

O Inpe, criado na década de 1960, tem como missão produzir ciência e tecnologia nas áreas espacial e do ambiente terrestre e oferecer produtos e serviços singulares em benefício do Brasil. Com mais de mil servidores, atua nas áreas de meteorologia e mudanças climáticas, observação da Terra, ciências espaciais e atmosféricas e engenharia espacial. $\mathrm{O}$ Inpe possui também laboratórios associados em computação aplicada, combustão e propulsão, física de materiais e de plasmas. O instituto presta serviços operacionais de previsão do tempo e clima, monitoramento do desmatamento da Amazônia Legal, rastreio e controle de satélites, medidas de queimadas, raios e poluição do ar, além de realizar testes e ensaios industriais de alta qualidade.

O CTI, por sua vez, atua desde o início da década de 1980 com a missão de gerar, aplicar e disseminar conhecimentos em tecnologia da informação, em articulação com os agentes socioeconômicos, promovendo inovações que atendam às necessidades da sociedade. Seus principais focos de atuação são as áreas de componentes eletrônicos, mostradores de informação, microeletrônica, sistemas, software e aplicações de TI, como robótica, softwares de suporte à decisão e tecnologias 3D para indústria e medicina. Organizado em 12 laboratórios, com cerca de 280 pesquisadores e sediado em Campinas (também no estado de São Paulo), tem grande foco na geração de inovações em bens e serviços de TI, por meio de parcerias com o setor industrial e prestação de serviços tecnológicos.

A seguir apresenta-se a análise comparada para os cinco temas gerenciais utilizados neste artigo. A análise compõe evidências encontradas tanto na pesquisa documental quanto na aplicação presencial do questionário e são aqui apresentadas de forma conjunta.

\subsubsection{Governança institucional}

Nessa temática de governança examinam-se os espaços gerenciais relacionados com mecanismos tais como: existência e tipo de ação de conselhos consultivos, com ênfase nos conselhos técnico-científicos; formas de organização e funcionamento das diretorias; formas e processos de indicação de chefias e cargos internos de liderança; organograma; e formas e mecanismos de comunicação. 
$\mathrm{Na}$ análise sobre instâncias consultivas, pode-se verificar que os dois institutos têm Conselhos Técnico-Científicos (CTCs), conforme determinação do MCTI. O ponto crítico a observar, além da existência em si desses conselhos e das diferenças de forma e funções entre eles, é o da maior ou menor importância que o instituto confere ao papel desse órgão em sua estrutura e em suas decisões estratégicas.

Nos casos dos dois IPPs estudados as funções desses conselhos são semelhantes: validar o relatório de gestão apresentado para o MCTI; avaliar os resultados de projetos e atividades dos institutos; acompanhar a gestão de RH; supervisionar a política de C\&T; entre outras. São também semelhantes as composições, embora haja diferenças na participação de membros internos e externos. Em ambos os casos quem preside os CTCs são os diretores das instituições. Este último ponto já revela uma prática não muito ortodoxa de governança. Como decorrência ou não desse modelo, o fato é que em nenhum dos casos analisados o CTC assume importância estratégica, muito embora não haja qualquer impeditivo do modelo jurídico para que isso ocorra. Essa evidência é também relatada na literatura como predominante, como demonstrado por Ocde (2011), Arnold (2007) e Bin e colaboradores (2013).

Tratando agora das formas de organização da direção dos institutos, foram também encontradas semelhanças e diferenças. ${ }^{6} \mathrm{Na}$ gestão que esteve à frente do Inpe entre 2005 e 2012 foram implantados uma Diretoria Colegiada e um Comitê de Programas, sendo essas estruturas formalmente definidas como base de um modelo gerencial parcialmente descentralizado. ${ }^{7}$ Outros modelos, colegiados e não colegiados, já foram implantados no instituto em outros momentos, mostrando um espaço de autonomia nesse quesito de governança.

No CTI, embora no período analisado a direção da instituição tenha se mantido nos padrões hierárquicos tradicionais, mais centralizados, organizaram-se Comitês Temáticos, como forma de tornar a gestão mais participativa. No modelo de gestão do Inpe também se encontraram Comitês Assessores similares aos comitês temáticos do CTI, com função de compartilhamento e comunicação entre as áreas finalísticas e entre estas e a direção do Instituto. Esse ponto demonstra razoável autonomia do IPP para definir seu modelo de gestão. Outro ponto essencial da governança é o processo de escolha de chefias internas. O Inpe se utiliza de formas variadas: comitês de busca, eleição e indicação pelo diretor, diferentemente do CTI, que se utiliza exclusivamente da indicação. ${ }^{8}$ Como se sabe, a escolha de chefias é um elemento crítico de modelos gerenciais. Características como liderança, dedicação, conhecimento técnico, experiência podem ser identificadas de diversas maneiras e certamente a forma pela qual as chefias são formalizadas em cargos interfere nos resultados da Instituição. Os IPPs da

\footnotetext{
${ }^{6}$ Embora o responsável legal do IPP da ADF seja sempre o diretor-geral, nada impede que haja modelos de governança mais descentralizados ou participativos. Essa é uma decisão da própria instituição, particularmente do seu diretor. ${ }^{7}$ Diz-se parcialmente porque um modelo totalmente descentralizado seria, no limite, um amplo colegiado com participação de chefes e representantes de todas as unidades do instituto. Também não é centralizado porque as decisões de uma diretoria colegiada são tomadas coletivamente e não exclusivamente pelo diretor-geral.

${ }^{8}$ Também há possibilidades de haver indicação política vinda do governo, mesmo para segundo e terceiro escalões.
} 
administração federal têm a liberdade de estabelecer as formas gerenciais mais convenientes de seleção de seus líderes internos.

A autonomia para compor os próprios organogramas, outro elemento do modelo de governança, é, na administração direta, naturalmente limitada pelos padrões utilizados pelo governo federal. Nos casos estudados, ambos IPPs assumem formas divisionais, hierárquicas, com forte influência do tipo e número de cargos comissionados disponíveis para a instituição.

Apesar dessa limitação, nada impede a adoção de outros modelos, como esquemas matriciais que combinem coordenação de programas com coordenação de departamentos ou divisões. Estruturas diversas em diferentes IPPs da administração direta já foram relatadas por outros autores (Garcia e Salles-Filho, 2009).

A comunicação dos institutos se diferencia na questão da estruturação interna: o Inpe conta com uma área de gestão da comunicação (com normas e procedimentos) e uma área de assessoria de imprensa que tem a responsabilidade de fazer a comunicação externa. No CTI, essas funções estão integradas com outras que não são específicas da área de comunicação. Com relação à comunicação interna, não foi encontrada nenhuma diferença significativa entre os casos estudados, embora seja esse um espaço de livre opção por parte dos IPPs.

Já a comunicação externa é uma atividade parcialmente controlada pelo MCTI. Apesar desse controle, encontram-se comportamentos flexíveis (criação de centro de visitantes, material de divulgação para eventos diversos - feiras, congressos etc.) das partes envolvidas (governo e IPP). De toda maneira, a influência política do governo (uma decorrência do modelo de $\mathrm{ADF}$ ) tende a limitar a comunicação mais pelo conteúdo do que pela forma, havendo liberdade para a criação de políticas mais ou menos sistemáticas de comunicação institucional. Os dois estudos da Ocde aqui referenciados (Ocde, 2003, 2011) apontam o tema da comunicação como central para o ambiente crescentemente competitivo da pesquisa em geral e dos IPPs em particular.

\subsubsection{Ciclo completo do planejamento}

Os dois institutos realizam planejamento estratégico, conforme determinação do MCTI. Na elaboração do planejamento estratégico há mais similaridades do que diferenças. Uma das diferenças está na contratação de consultorias externas para conduzir o planejamento (caso do Inpe em 2005 - a consultoria conduziu todo o processo de planejamento estratégico, que resultou na elaboração do Plano Diretor). No CTI, o processo tem sido conduzido internamente, muito embora se busque, como no Inpe, representatividade de todas as áreas com vistas a um maior comprometimento em termos de execução das diretrizes apontadas.

O CTI tem se utilizado de ferramentas de prospecção tecnológica e monitoramento de demandas de forma sistemática, o que, segundo os entrevistados, estaria ajudando o desempenho em termos de captação de recursos externos. O Inpe, apesar de já ter utilizado ferramentas dessa natureza, não possui um esforço sistemático e institucional de prospecção. 
O Inpe utiliza ferramentas de gestão de portfólio e projetos para algumas áreas, com destaque para o desenvolvimento de satélites. O CTI, por sua vez, utiliza o Sistema de Gestão Tecnológica (Sigtec), ferramenta desenvolvida internamente pela instituição, que tem como finalidade a gestão dos seus projetos e atividades. Nos dois institutos, o principal foco de gestão são os projetos determinados pelo Plano Plurianual (PPA). Em quaisquer dos casos seria possível a introdução de ferramentas avançadas de gestão de portfólio baseadas em business intelligence ou em Big Data.

Com relação à avaliação de resultados, ambos prestam contas para o MCTI e são auditados pelo TCU. Parte dos indicadores do TCG é comum entre o Inpe e o CTI (uma vez que o Ministério utiliza referências comuns para realização de comparações históricas do desempenho entre os institutos) e parte é específica, negociada pelo IPP junto ao MCTI.

Entretanto, nenhum dos dois institutos avançou na direção de criar um modelo de avaliação de impactos e de balanço social, como faz a Embrapa, ou formato congênere. Esse tipo de prática, quando realizada, tem forte poder de comunicação institucional e serve como documento de divulgação e negociação do instituto com a sociedade e seus diversos segmentos. Desde o final dos anos 1990 e do início dos anos 2000, Arnold e colaboradores (1998) e Ocde (2003) já apontavam o tema do planejamento de ciclo completo, incluindo a avaliação de impactos como fundamental na reorganização de IPPs.

\subsubsection{Manutenção e ampliação de recursos humanos}

Tanto o Inpe quanto o CTI contratam por meio de concurso público e possuem em seu quadro de pessoal, além dos servidores públicos, bolsistas de agências de fomento, estagiários e pessoal terceirizado (via contratos temporários e via fundações), em consonância com a tendência verificada para os IPPs em âmbitos nacional e internacional.

A contratação por concurso público é um processo extremamente moroso. Hoje, para ambos os institutos, os prazos de contratação não ficam abaixo de um ano, entre publicar o edital, realizar o concurso e finalizar a contratação. Ademais, a possibilidade de abertura de concurso depende de autorização do próprio MCTI, que por sua vez depende de autorização do MPOG. Novos concursos têm sido realizados em média a cada quatro anos.

Os espaços de flexibilidade, no caso de recursos humanos, ficam por conta de alternativas de contratação temporária, bolsas e projetos via fundações de apoio. Trata-se de expedientes que se tornaram regra nos IPPs. Os dois casos aqui apresentados não são exceção. Durante parte do período aqui analisado, o CTI chegou a contar com mais pessoal contratado via Fundação que servidores concursados.

O Inpe, proibido em meados dos anos 2000 de contratar pessoal via Fundação, passou a usar o Decreto n 4.748/2003 que autoriza concurso simplificado para cargos por período determinado, contando hoje com 118 servidores contratados nesse modelo. Entretanto, em decisão recente do TCU, esses servidores terão que ser agora demitidos porque o entendimen- 
to foi de que não se pode contar com pessoal assim contratado para atividades finalísticas (entenda-se pesquisa).

As restrições do modelo jurídico, combinadas com restrições fiscais e orçamentárias do governo federal, além das ingerências políticas, formam, no caso de recursos humanos, um obstáculo concreto para a boa gestão dos IPPs da ADF. Os espaços de autonomia e flexibilidade existem, mas são limitados e arriscados para o gestor público, não porque descumprem a Lei, mas porque as interpretações dos órgãos de controle, ao se manifestarem de forma diferente, podem revelar problemas ex post.

Como se percebe, não são exatamente espaços de autonomia, mas expedientes necessários para o próprio cumprimento das missões institucionais. Sem renovação e ampliação de massa crítica, a capacidade de competir nos sistemas de ciência e tecnologia tende a declinar. ${ }^{9}$

Nos dois institutos há iniciativas para a capacitação de pessoal, que podem ser realizadas interna ou externamente e que podem incluir, em alguns casos, os terceirizados, estagiários e bolsistas, além dos servidores. Enquanto no Inpe há avaliações sistemáticas dessas iniciativas de capacitação, além de incentivos para que os servidores partilhem os conhecimentos adquiridos ministrando cursos internos, no CTI não há esta prática.

O Inpe e o CTI realizam avaliação de desempenho individual, conforme determina a legislação e nos moldes previstos pelo MCTI, muito embora o CTI já tenha implantado o novo modelo do MCTI aprovado em 2012, e o Inpe não. Nenhum dos dois institutos utiliza outros componentes de modelos de avaliação de desempenho individual (como avaliação de chefias, por exemplo). Ademais, o Inpe tem trabalhado o desenvolvimento de lideranças, gestão por competências e análise de clima organizacional, não havendo nenhuma iniciativa por parte do CTI nesses assuntos Esses instrumentos podem, portanto, ser amplamente desenvolvidos pelos IPPs da ADF.

\subsubsection{Captação e gestão de recursos financeiros}

Esse tema refere-se especialmente à autonomia e flexibilidade para uso dos recursos orçamentários e para captação e administração de recursos extraorçamentários. A gestão dos recursos orçamentários é um dos itens nos quais há muito pouco espaço de autonomia e flexibilidade para os IPPs da ADF. Já a captação de recursos extraorçamentários encontra razoável autonomia nos IPPs.

Seria então natural que as diferenças encontradas entre os institutos fossem pequenas. Entretanto, também nesse tema, há diferenças importantes.

A gestão de compras é um dos pontos de maior entrave que os institutos encontram, em função das restrições da Lei no 8.666/1993 e também da interpretação e das formas de fisca-

\footnotetext{
${ }^{9}$ A nova legislação de fundações de apoio (Lei no 12.863 , de 24 de setembro de 2013) normatiza a contratação temporária de pessoal via fundação, trazendo certo alento para os IPPs, embora não resolvendo a questão fundamental da necessidade de vagas e de planos de carreira e desenvolvimento compatíveis com o mercado de trabalho.
} 
lização dos órgãos de controle. Hoje os dois institutos contam com um número reduzido de pessoal na área de compras, não têm uma área jurídica interna que dê suporte aos processos licitatórios e estão submetidos a um órgão jurídico externo, a Consultoria Jurídica da União (CJU), embora possam contar com alguma assessoria interna para fazer a ligação com a CJU.

Se for feito um estudo sobre os passos processuais para execução de orçamento de capital e custeio (exceto pessoal) dos 14 IPPs de administração direta do MCTI, serão encontrados 14 procedimentos diferentes, com diferentes números e tipos de passos e autorizações internos para cada IPP. A otimização interna é assim possível, desejável e factível, sem infringir o marco legal.

Os casos aqui apresentados não fogem à regra. O uso de instrumentos tais como adesão às atas de registros de preços e estabelecimento de relação com órgãos de controle são os mecanismos utilizados pelo CTI para conseguir melhorar seu índice na execução do orçamento, que tem estado em torno de 85\% na média do período de 2007 a 2011 . Já o Inpe, por diversas razões, tem alcançado índices menores, na faixa dos 65\% no mesmo período. Parte dessas diferenças deve-se a mecanismos e complexidades internas aos IPPs.

Simplificação de passos internos e especialização de pessoal responsável por licitações e permanente atualização de procedimentos são mecanismos necessários e possíveis de implementar.

Esse é, juntamente com o tema dos recursos humanos, um dos elementos do modelo gerencial mais afetado pelo modelo jurídico dos IPPs federais no Brasil. Mesmo com esforços internos de otimização, os tempos médios de licitações, tanto no Inpe quanto no CTI, ficam entre quatro e seis meses. Esse é um dos temas que mais ameaça a competitividade dos IPPs em face do crescentemente competitivo mundo da P\&D. ${ }^{10}$

Quanto à capacidade de captação de recursos extraorçamentários, o CTI tem captado no período entre $20 \%$ e $30 \%$, enquanto o Inpe tem ficado na faixa dos $15 \%$ a $23 \%$. Esses recursos são oriundos de leis de incentivo fiscal, agências de fomento e projetos com empresas privadas. Essas diferenças estão diretamente ligadas ao modelo gerencial e à criação interna de competências. A forma pela qual atuam as fundações de apoio também ajuda a explicar esse fenômeno, sendo algumas mais agressivas que outras na busca por recursos. Nenhum dos dois institutos tem pessoal capacitado e designado para captação de recursos extraorçamentários, nem para gerenciamento especializado de contratos com empresas.

Na literatura, o tema da captação de recursos é apontado por Arnold (2007), Boden e colaboradores (2006), Jansen (2007) e Ocde (2011) como aquele que vem trazendo maior impacto nos processos de reorganização de IPPs, na medida em que o esforço de financiamento abre caminhos para a ampliação de contratos de prestação de serviços com diferentes

\footnotetext{
${ }^{10}$ A nova legislação das fundações de apoio (Lei no 12.863 , de 24 de setembro de 2013) abre possibilidades de autonomia e flexibilidade para as IPPs na medida em que autoriza essas fundações a assumir a gestão parcial de compras de bens e serviços ligados diretamente a atividades de CTI. Entretanto, a lei também deixa claro que os recursos públicos recebidos pelas fundações de apoio sofrerão controle finalístico pelo órgão de controle governamental competente.
} 
fontes e finalidades. Ao mesmo tempo, a necessidade de financiamento da pesquisa encontra fontes competitivas para o que os IPPs devem estar mais e mais preparados.

\subsubsection{Relacionamento com atores dos sistemas de inovação}

Os dois institutos têm atuado de forma ativa em redes de cooperação e no estabelecimento de parcerias. A participação nessas iniciativas pode vir por iniciativa do instituto, do pesquisador individual (ou de um grupo) ou por determinação do MCTI, devido ao direcionamento de certas políticas ou acordos firmados com outros países.

O CTI possui uma unidade específica que realiza desenvolvimento de mercado, com o intuito de auxiliar empresas, governos e outras instituições a acessar as competências do centro, assim como o estabelecimento de parcerias para o desenvolvimento conjunto. No Inpe não há ações dessa natureza estruturadas, exceto em duas de suas unidades: o Laboratório de Integração e Testes (LIT) e a Coordenação de Observação da Terra (OBT).

Tanto o Inpe quanto o CTI têm Núcleos de Inovação Tecnológica (NIT) internos e participam da Rede Mantiqueira (rede regional de NITs estabelecida pelo MCTI). Também os dois institutos têm políticas e procedimentos definidos para o patenteamento e transferência de conhecimento.

As leis de fomento a inovação são parcialmente exploradas pelos institutos. O CTI tem se utilizado com frequência da Lei de Informática, captando recursos extraorçamentários. O Inpe procura utilizar o instrumento de encomenda pública junto às empresas. Em ambas as organizações há vários exemplos de forte interação com outros atores públicos e privados. O CTI relaciona-se diretamente com a indústria de tecnologia de informação e comunicação, além de ter ações com governo e entidades de representação setorial. É um braço de atuação da Secretaria de Política de Informática (Sepin) do MCTI, conduzindo vários programas de governo. O Inpe, por sua vez, é o braço executivo do programa brasileiro de satélites e é também um centro de referência em previsão do tempo e estudos de clima, observação da Terra, mensuração do desmatamento e de ocorrências de fenômenos naturais extremos. Ambos interagem com empresas, seja encomendando, transferindo ou desenvolvendo tecnologias conjuntamente.

Nesse tema, o modelo jurídico deixa razoável espaço de autonomia e flexibilidade para o IPP. O maior entrave está no uso da encomenda pública. O instrumento da encomenda pública é dos mais relevantes para alavancagem da inovação tecnológica, inclusive com participação de IPPs (Miles e Rigby, 2013), e nesse particular o marco jurídico do setor público brasileiro ainda coloca limitações severas, fazendo com que os institutos tenham que buscar alternativas criativas para a encomenda de partes e sistemas essenciais ao desenvolvimento de tecnologias mais sofisticadas.

Em resumo, as análises anteriores permitem afirmar que tanto o Inpe quanto o CTI têm aproveitado de forma heterogênea as margens de autonomia e flexibilidade que derivam de seu modelo jurídico e que esse aproveitamento está essencialmente ligado às decisões da alta gerência dos institutos e à própria cultura institucional. 


\section{Conclusões}

Tanto a revisão de literatura quanto os dois estudos de caso e a análise documental realizados oferecem elementos para responder às questões de pesquisa colocadas no início deste artigo.

Respondendo à primeira questão, é possível dizer que os IPPs da administração direta no Brasil são efetivamente limitados em suas ações por conta de modelos jurídicos restritivos, particularmente nos casos de gestão orçamentária e financeira e de manutenção e ampliação de recursos humanos. Entretanto, essas limitações vêm sendo parcialmente contornadas por diversos mecanismos, entre os quais o uso de fundações de apoio e outros instrumentos, como otimizações internas de processos operacionais, aproximação com órgãos de controle e uso crescente de recursos extraorçamentários.

Já nos temas de governança institucional, ciclo completo do planejamento e relação com atores do SI, as limitações são menores, abrindo oportunidades de inovações gerenciais e organizacionais que podem ter impactos positivos sobre o cumprimento das missões institucionais.

É possível também afirmar, respondendo à segunda questão, que as inovações gerenciais e organizacionais em IPPs da administração direta podem levar à criação de gestões sensivelmente diferenciadas, mostrando que há espaços consideráveis que podem impactar no desempenho das instituições. Entretanto, para responder completamente à questão (se há modelos mais ou menos eficientes), é preciso avançar com novas pesquisas e comparar modelos gerenciais e organizacionais com indicadores de desempenho dos IPPs. Essa é ainda uma tarefa em aberto e um tema central da agenda de pesquisa nessa temática.

Sobre a terceira questão, se são necessários regimes jurídicos alternativos para a competitividade dos IPPs, a pesquisa oferece elementos para a formulação de uma resposta, se não categórica, ao menos bem fundamentada. Tendo em vista as dificuldades relacionadas a recursos humanos e financeiros e tendo em vista que o ambiente da pesquisa está cada vez mais competitivo, com novos atores que podem apresentar competências semelhantes às dos IPPs com menores custos de transação, maior agilidade relacional e flexibilidade operacional, conforme mostrado na revisão de literatura, a resposta a essa questão é positiva.

As alternativas gerenciais e organizacionais dentro do regime jurídico da ADF, embora importantes e passíveis de serem exploradas, são relativamente limitadas e podem, no médio e longo prazo, comprometer o desempenho dessas organizações, conforme vêm atestando vários dos estudos citados neste artigo.

Muito embora a transformação dos modelos jurídicos tenha estado no foco do debate sobre o futuro dos IPPs (no mundo e no Brasil), as efetivas transformações pelas quais estas organizações têm passado (e provavelmente parte importante dos resultados que têm logrado) devem muito à busca de inovações gerenciais e organizacionais.

A criação de modelos jurídicos alternativos aos atuais pode por certo impactar esse processo, impulsionando trajetórias gerenciais mais robustas e revigorando a importância dos IPPs no sistema de ciência, tecnologia e inovação. Entretanto, de nada adiantam modelos jurídicos mais flexíveis se não forem acompanhados de inovações gerenciais e organizacionais compatíveis. 
Os casos da gestão de recursos orçamentários e humanos e da flexibilidade em compras são particularmente evidentes e precisam de novos marcos regulatórios porque dificultam o cumprimento das missões e colocam em risco a própria relevância dessas instituições quando se vislumbra um cenário de internacionalização e globalização da pesquisa.

Todavia, a mudança no modelo jurídico de forma isolada não garantirá melhor desempe-

nho desses institutos. É preciso um esforço de profissionalização e de permanente atualização dos modelos gerenciais e organizacionais. Os IPPs precisam criar elites gerenciais e promover programas internos de capacitação e desenvolvimento gerencial que lhes permita enfrentar sistemas cada vez mais competitivos no mundo da ciência, da tecnologia e da inovação.

O levantamento de outras experiências, envolvendo outros modelos jurídicos, pode certamente complementar as conclusões aqui evidenciadas permitindo uma ampliação da discussão sobre a adequação de modelos jurídicos para os IPPs e os indicativos para que essas organizações construam modelos gerenciais mais exitosos no futuro.

\section{Referências}

ADLER, Niclas; ELMQUIST, Maria; NORRGREN, Flemming. The challenge of managing boundary-spanning research activities: experiences from the Swedish context. Research Policy, v. 38, n. 7, p. 1136-1149, 2009.

ALBUQUERQUE, Rui H. et al. O setor público de pesquisa agrícola no estado de São Paulo. Caderno de Difusão Tecnológica, Brasília, v. 3, n. 1, p. 79-132, jan./abr. 1986.

ALVES, Eliseu. Modelo institucional da Embrapa. In: YEGANIANTZ, Levon (Org.) Perspectiva histórica e desenvolvimento institucional. Brasília: Embrapa, 1985. p. 373-393.

ARNOLD, Eric et al. Strategic planning in research and technology institutes. R\&D Management, v. 28, n. 2, p. 89-100, 1998.

ARNOLD, Natalie. The application of the concept of governance to the structures of German extra-university research organizations from a legal perspective. In: JANSEN, Dorothea (Ed.). New forms of governance in research organizations. Dordrecht: Springer, 2007. p. 177-188.

ÅSTRÖM, Tomas et al. International comparison of five institute systems. Kobenhavn: Forsknings- og Innovationsstyrelsen, 2008.

BIN, Adriana et al. Organization of research and innovation: a comparative study of public agricultural research institutions. Journal of Technology Management \& Innovation, v. 8, n. Special Issue Altec, p. 209-218, 2013.

BODEN, Rebecca et al. The appliance of science? New public management and strategic change. Technology Analysis \& Strategic Management, v. 18, n. 2, p. 125-141, 2006.

BRUM, José A.; MENEGHINI, Rogério. O Laboratório Nacional de Luz Síncrotron. São Paulo em Perspectiva, v. 16, n. 4, p. 48-56, 2002. 
CGEE. Centro de Gestão e Estudos Estratégicos. Modelos institucionais das organizações de pesquisa. Brasília: CGEE, 2010.

COCCIA, Mario; ROLFO, Sccondo. Strategic change of public research units in their scientific activity. Technovation, v. 28, n. 8, p. 485-494, 2008.

DOSSA, Alvaro A.; SEGATTO, Andréa P. Pesquisas cooperativas entre universidades e institutos públicos no setor agropecuário brasileiro: um estudo de caso. Rev. Adm. Pública, v. 44, n. 6, p. 1327-1352, nov./dez. 2010.

FURTADO, André. Novos arranjos produtivos, estado e gestão da pesquisa pública. Ciência e Cultura, v. 57, n. 1, p. 41-45, 2005.

GARCIA, Ana E. B.; SALLES-FILHO, Sergio L. M. Trajetória institucional de um instituto público de pesquisa: o caso do Ital após 1995. Rev. Adm. Pública, v. 43, n. 3, p. 661-693, 2009.

GODOY, Arilda S. Introdução à pesquisa qualitativa e suas possibilidades. Revista de Administração de Empresas, São Paulo, v. 35, n. 2, p. 57-63, mar./abr. 1995a.

GODOY, Arilda S. Pesquisa qualitativa: tipos fundamentais. Revista de Administração de Empresas, São Paulo, v. 35, n. 3, p. 20-29, mai./jun. 1995b.

HALL, Andy. Public-private sector partnerships in an agricultural system of innovation: concepts and challenges. International Journal of Technology Management and Sustainable Development, v. 5, n. 1, p. 3-20, 2006.

HOLLANDERS, Hugo; SOETE, Luc. O crescente papel do conhecimento na economia global. In: ORGANIZAÇÃO DAS NAÇÕES UNIDAS. Relatório Unesco sobre ciência 2010: o atual status da ciência em torno do mundo: resumo executivo. Brasília: Unesco do Brasil, 2010. p. 5-31.

JAIN, Ravi K.; TRIANDIS, Harry C. Management of research and development organizations: managing the unmanageable. 2. ed. Nova York: John Wiley \& Sons, Inc., 1997.

JANSEN, Dorothea. Governance of research - working towards interdisciplinary concepts. In: JANSEN, Dorothea. (Ed.). New forms of governance in research organizations. Dordrecht: Springer, 2007. p. 109-136.

LAREDO, Phillipe; MUSTAR, Phillipe. Public sector research: a growing role in innovation systems. Minerva, v. 42, p. 11-27, 2004.

MADI, Luis F.; SALLES-FILHO, Sergio L. M.; ALBUQUERQUE, Rui H. P. L. Uma dupla agenda para o futuro da agricultura paulista. Campinas: APTA (Agência Paulista de Tecnologia dos Agronegócios), 2006.

MATTOS, Paulo T. L.; ABDAL, Alexandre. Estados Unidos: mudanças jurídico-institucionais e inovação. In: ARBIX, Glauco et al. (Org.). Inovação: estratégia de sete países. Brasília: ABDI, 2010. p. 92-120. (Série Cadernos da Indústria ABDI, v. XV).

MEIRELLES, Hely L.; ALEIXO, Délcio B.; BURLE FILHO, José E. Direito administrativo brasileiro. São Paulo: Malheiros Editores Ltda, 2012. 
MILES, Ian; RIGBY, John. Demand-led innovation. In: COX, Deborah; RIGBY, John. Innovation policy challanges for the $21^{\text {st }}$ century. Nova York: Routledge, 2013. p. 6-33.

OECD. Governance of public research: toward better practices. Paris: Oecd Publications Service, 2003. OECD. Public research institutions: mapping sector trends. Paris: Ocde, 2011.

PEREIRA, Julio C. R. Análise de dados qualitativos. São Paulo: Edusp, 1999.

POTI, Bianca; REALE, Emanuela. Convergence and differentiation in institutional change among European public research systems: the decreasing role of public research institutes. Science and Public Policy, v. 27, n. 6, p. 421-431, 2000.

PREST. A comparative analysis of public, semi-public and recently privatised research centres. Final Project Report, Part 1: Summary Report, 2002.

QUENTAL, Cristiane et al. O papel dos institutos públicos de pesquisa na inovação farmacêutica. Rev. Adm. Pública, v. 35, n. 5, p. 135-161, 2001.

ROSA, Marcio F. E. Direito administrativo. 9. ed. São Paulo: Saraiva, 2007. v. 19.

SALLES-FILHO, Sergio L. M.; BONACELLI, Maria B. Trends in the organization of public research institutions: lessons from the Brazilian case. Science and Public Policy, v. 37, n. 3, p. 193-204, 2010.

SALLES-FILHO, Sergio L. M. et al. Ciência, tecnologia e inovação: a reorganização da pesquisa pública no Brasil. Campinas: Komedi, 2000.

SALLES-FILHO, Sergio L. M. et al. Metodologia para o estudo da reorganização institucional da pesquisa pública: o índice de modernização institucional. Revista Parcerias Estratégicas, v. 1, n. 9, p. 86-108, 2000.

SENKER, Jacqueline. Introduction to a special issue on changing organization and structure of European public-sector research systems. Science and Public Policy, v. 27, n. 6, p. 394-396, 2000.

SLATYER, Ralph O. Cooperative research centres: the concept and its implementation. Higher Education, v. 28, n. 1, p. 147-158, jul. 1994.

VONORTAS, Nicholas. Technology policy in the United States and the European Union: shifting orientation towards technology users. Science and Public Policy, v. 27, n. 2, p. 97-108, 2000.

Valéria Cristina dos Santos Ribeiro é doutora em política científica e tecnológica pela Universidade Estadual de Campinas (Unicamp) e analista em C\&T no Instituto Nacional de Pesquisas Espaciais (Inpe). E-mail: valeria.ribeiro@inpe.br.

Sergio Luiz Monteiro Salles-Filho é professor titular do Departamento de Política Científica e Tecnológica, Instituto de Geociências, Universidade Estadual de Campinas (Unicamp).E-mail: sallesfi@ige.unicamp.br.

Adriana Bin é professora doutora na Faculdade de Ciências Aplicadas, Universidade Estadual de Campinas (Unicamp). E-mail: adriana.bin@fca.unicamp.br. 